\title{
Kinetic-Scale Turbulence in the Venusian Magnetosheath
}

T. A. Bowen ${ }^{1}$, S. D. Bale ${ }^{1,2}$, R. Bandyopadhyay ${ }^{3}$, J.W. Bonnell ${ }^{1}$, A. Case $^{4}$, A. Chasapis, ${ }^{5}$, C. H. K. Chen ${ }^{6}$, S. Curry ${ }^{1}$, T. Dudok de Wit ${ }^{7}$, K. Goetz ${ }^{8}$, K.

Goodrich $^{1}$, J. Gruesbeck ${ }^{9}$, J. Halekas ${ }^{10}$, P. R. Harvey ${ }^{1}$, G. G. Howes ${ }^{10}$, J.C. Kasper $^{11}$, K. Korreck ${ }^{4}$, D. Larson ${ }^{1}$, R. Livi ${ }^{1}$, R. J. MacDowall ${ }^{9}$, D. M. Malaspina $^{5,12}$, A. Mallet $^{1}$, M.D. McManus ${ }^{1}$,B. Page ${ }^{1}$, M. Pulupa ${ }^{1}$, N. Raouafi $^{13}$, M.L. Stevens ${ }^{4}$, P. Whittlesey ${ }^{1}$

${ }^{1}$ Space Sciences Laboratory, University of California, Berkeley, CA 94720-7450, USA ${ }^{2}$ Physics Department, University of California, Berkeley, CA 94720-7300, USA

${ }^{3}$ Department of Astrophysical Sciences, Princeton University, Princeton, NJ 08544, USA ${ }^{4}$ Smithsonian Astrophysical Observatory, Cambridge, MA 02138 USA

${ }^{5}$ Laboratory for Atmospheric and Space Physics, University of Colorado, Boulder, CO 80303, USA

${ }^{6}$ School of Physics and Astronomy, Queen Mary University of London, London E1 4NS, UK

${ }^{8}$ LPChool of Physics and Astronomy, University of Minnesota, Minneapolis, MN 55455

${ }^{9}$ Planetary Magnetospheres Laboratory, NASA Goddard Space Flight Center, Greenbelt, MD 20771, USA

${ }^{10}$ Department of Physics and Astronomy, University of Iowa, Iowa City, IA 52242, USA

${ }^{11}$ Climate and Space Sciences and Engineering, University of Michigan, Ann Arbor, MI 48109, USA

${ }^{12}$ Astrophysical and Planetary Sciences Department, University of Colorado, Boulder, CO, USA 13 Johns Hopkins University Applied Physics Laboratory, Laurel, MD

Corresponding author: Trevor A. Bowen, tbowen@berkeley.edu

This article has been accepted for publication and ${ }^{-1}$ undergone full peer review but has not been through the copyediting, typesetting, pagination and proofreading process, which may lead to differences between this version and the Version of Record. Please cite this article as doi: 10.1029/2020GL090783. 


\begin{abstract}
While not specifically designed as a planetary mission, NASA's Parker Solar Probe (PSP) mission uses a series of Venus gravity assists (VGAs) in order to reduce its perihelion distance. These orbital maneuvers provide the opportunity for direct measurements of the Venus plasma environment at high cadence. We present first observations of kinetic scale turbulence in the Venus magnetosheath from the first two VGAs. In VGA1, PSP observed a quasi-parallel shock, $\beta \sim 1$ magnetosheath plasma, and a kinetic range scaling of $k^{-2.9}$. VGA2 was characterised by a quasi-perpendicular shock with $\beta \sim 10$, and a steep $k^{-3.4}$ spectral scaling. Temperature anisotropy measurements from VGA2 suggest an active mirror mode instability. Significant coherent waves are present in both encounters at sub-ion and electron scales. Using conditioning techniques to exclude these electromagnetic wave events suggests the presence of developed sub-ion kinetic turbulence in both magnetosheath encounters.
\end{abstract}

\title{
1 Introduction
}

Astrophysical environments are often characterized by nonlinear turbulent processes, which transfer energy from large fluid-like scales to kinetic dissipative scales. The relative accessibility of space-plasma environments has driven our understanding of these universal processes (Bruno \& Carbone, 2005; Chen, 2016; Verscharen et al., 2019). While properties of large scale magnetohydrodynamic (MHD) turbulence have been studied since the earliest days of space exploration (Coleman, 1968; Matthaeus \& Goldstein, 1982), relatively recent advancements in instrumentation have enabled analysis of kinetic scale turbulence (Leamon et al., 1998; Alexandrova et al., 2012; Chen \& Boldyrev, 2017).

Evidence for kinetic scale plasma-turbulence largely stems from observations of the terrestrial magnetosphere and solar wind. At ion kinetic scales magnetic spectra steepen, due to some combination of dispersive and dissipative effects, leading to a subion scale energy cascade (Alexandrova et al., 2008; Sahraoui et al., 2009; Alexandrova et al., 2009; Sahraoui et al., 2010; Alexandrova et al., 2012). Kinetic spectra with approximate $k^{-2.7}$ scaling characterize the solar wind at $1 \mathrm{AU}$ and the inner heliosphere (Sahraoui et al., 2009; Chen et al., 2010; Alexandrova et al., 2012; Sahraoui et al., 2013; Bowen, Mallet, Bale, et al., 2020). The observed steepening is consistent with the dispersion of Alfvénic to kinetic Alfvén wave (KAW) turbulence alongside some intermittency or dissipation (Schekochihin et al., 2009; Boldyrev \& Perez, 2012a; Howes et al., 2011; Chen et al., 2013; Franci et al., 2015, 2016). At electron kinetic scales, further spectral steepening is measured (Alexandrova et al., 2009, 2012; Sahraoui et al., 2013; Huang et al., 2014; Chen \& Boldyrev, 2017).

Kinetic scale steepening in Earth's magnetosphere (Dudok de Wit \& Krasnoselkikh, 1996; Czaykowska et al., 2001) is likely connected to magnetospheric heating (Sundkvist et al., 2007); however the shape and spectral scaling of magnetospheric turbulence is a topic of significant debate. Commonly observed inertial range turbulence, with approximate Kolmogorov-like $k^{-5 / 3}$ scaling, is not universally present in the terrestrial magnetosphere (Czaykowska et al., 2001; Alexandrova et al., 2008); a common interpretation is that shock structure may prevent the formation of fluid scale turbulence in the magnetosheath (Vörös, Zhang, Leubner, et al., 2008; Huang et al., 2017; Chhiber et al., 2018). However, instabilities may serve as a source of turbulent and nonlinear fluctuations, which may vary the inertial range spectrum (Sahraoui et al., 2006). Kinetic range spectra observed in the terrestrial magnetosphere are similar to the 1 au solar wind, consistent with KAW turbulence (Alexandrova et al., 2008; Huang et al., 2014; Chen \& Boldyrev, 2017). However, variation in kinetic range scaling of magnetosheath spectra has been reported (Rezeau et al., 1999; Alexandrova et al., 
2008; Huang et al., 2014), possibly attributable to intermittency (Alexandrova, 2008; Boldyrev \& Perez, 2012b; Zhao et al., 2016), or dissipation (Howes et al., 2011).

Knowledge of kinetic scale processes of extraterrestrial magnetospheres is limited by the resources required for distant space-missions. Saur (2004) suggest that turbulent dissipation is significant to heating Jupiter's magnetosphere. Saturn's magnetosphere has kinetic turbulence with scalings similar to that observed at Earth and inferred turbulent dissipation rates that can account, for magnetospheric heating (von Papen et al., 2014). Hadid et al. (2015) suggest that perpendicular shock geometry may prevent formation of an inertial range at Saturn, though kinetic scales are largely invariant behind both quasi-parallel and quasi-perpendicular shocks. Observations from Jupiter, reveal similar properties such as spectral steepening at kinetic scales, and the lack a $k^{-5 / 3}$ inertial range (Tao et al., 2015).

Kinetic-scale turbulence is also observed in the magetospheres of Mars and Mercury. Uritsky et al. (2011) study kinetic scale turbulence in Mercury's magnetosphere, observing a fluid-kinetic break, and steep anomalous scaling of inertial range fluctuations, attributed to finite Larmor radius (FLR) effects; the authors highlight potential ion-scale instabilities and the presence of coherent electron scale waves. Huang et al. (2020) suggest that no inertial range forms in Mercury's magnetosheath, and that heavy exospheric ions contribute to deviation from canonical $k^{-5 / 3}$ spectra. Ruhunusiri et al. (2017) demonstrate that spectral energy scaling of turbulence near Mars is well ordered by magnetospheric structure: shallow inertial range spectra are found in the magneosheath, though kinetic range turbulence seems developed; solar wind-like inertial range and kinetic spectra are observed near the magnetic pileup region, suggesting turbulent processing.

Parker Solar Probe utilizes resonant orbital encounters with Venus to reduce its perihelion altitude (Fox et al., 2016), providing an opportunity for detailed observations of kinetic scale turbulence in the Venusian magnetosphere. At closest approach, PSP will fly within $400 \mathrm{~km}$ of the Venusian surface, placing it within Venus's ionosphere (Zhang et al., 2007; Futaana et al., 2017). Though not designed specifically to study the Venus plasma environment, PSP shares technological heritage with modern magnetospheric missions (McFadden et al., 2008; Wygant et al., 2013; Kletzing et al., 2013). Observations made by PSP during these encounters promise to contribute significantly to understanding the planet's magnetosphere.

Nonlinear waves and MHD turbulence in Venusian plasma have been studied previously. Vörös, Zhang, Leubner, et al. (2008) demonstrate intermittent turbulence in the Venusian wake and magnetosheath. Based on observations of shallow spectra with Gaussian fluctuations, Vörös, Zhang, Leaner, et al. (2008) suggest that MHD turbulence may not develop uniformly throughout the magnetosphere, in agreement with observations from other planetary environments (Czaykowska et al., 2001; Hadid et al., 2015; Ruhunusiri et al., 2017; Huang et al., 2017; Chhiber et al., 2018). Xiao et al. (2018) show that shock geometry is important in shaping the inertial range, with developed $k^{-5 / 3}$ spectra appearing more readily behind quasi-parallel shocks. Xiao et al. (2020) additionally show that day/night asymmetry strongly affects the development of inertial scale turbulence. Many inertial scale nonlinear waves, instabilities, and vorticies have been reported near Venus, which are potential drivers of turbulence (Wolff et al., 1980; Amerstorfer et al., 2007; Balikhin et al., 2008; Pope et al., 2009; Walker et al., 2011; Golbraikh et al., 2013; Volwerk et al., 2016; Futaana et al., 2017).

There are relatively few kinetic scale observations of fluctuations at Venus. Dwivedi et al. (2015) suggest that a break exists between MHD and kinetic ranges, and that anomalous inertial range scaling is possibly due to mirror mode structures generated through temperature anisotropy. The authors suggest that kinetic scale fluctuations may be a combination of nonlinearly interacting kinetic turbulence with instability 
driven modes; however the observations are limited by the $1 \mathrm{~Hz}$ magnetometer resolution. Kinetic scale wave phenomenon have been studied in detail; with much focus on the Venusian ionosphere (Russell et al., 2013). High frequency, electron scale waves, likely generated through plasma instabilities, have been well documented in the foreshock, upstream solar wind, and magnetosheath (Strangeway, 2004). Ion scale waves have been identified both upstream and downstream the bow shock (Russell et al., 2006; Delva et al., 2015).

Here, we study signatures of kinetic scale turbulence in the Venusian magnetosheath. We demonstrate differences in spectral energy scalings in the kinetic range, likely due to bow-shock geometry, plasma $\beta$, and the presence of the mirror instability. In addition to kinetic scale turbulence, the sub-ion and electron scales in the magnetosheath are characterized by significant wave activity (Page, 2020). The use of conditioning (Sorriso-Valvo et al., 1999; Kiyani et al., 2006; Chen et al., 2014) to exclude coherent sub-ion scale waves reveals that despite significant differences in spectral scaling signatures of a developed kinetic cascade are present in both encounters. At electron scales the spectrum further steepens, similar to observations from Earth's magnetosphere (Huang et al., 2014; Chen \& Boldyrev, 2017).

\section{Data}

We implement measurements from the electromagnetic FIELDS instrument (Bale et al., 2016) as well as the Solar Wind Electron Alpha and Proton (SWEAP) investigation (Kasper et al., 2016) during PSP's first two Venus gravity assists (VGA1 occurring Oct 31, 2018 and VGA2 on Dec 26, 2019).

FIELDS measures electromagnetic fluctuations, creating a variety of data products (Bale et al., 2016; Malaspina et al., 2016; Pulupa et al., 2017; Bowen, Bale, et al., 2020). The magnetic field is measured by a low frequency fluxgate magnetometer (MAG) and an AC coupled search coil magnetometer (SCM). We use merged SCM and MAG (SCaM) data, with DC-146 Hz bandwidth (Bowen, Bale, et al., 2020). Following the first solar encounter, the SCM sensor $x$ axis has exhibited significant anomalous behavior. Thus, for VGA2 only two component magnetic field measurements (SCM $y$ and $z$ ) are available at kinetic scales.

PSP is specifically configured for measuring solar wind plasma in the inner heliosphere (Fox et al., 2016), which can complicate measurements of the Venusian plasma environment. During VGA1, the solar limb-sensor (which maintains correct pointing during solar encounters) responded to the Venusian albedo, turning off the instruments midway magnetospheric transit, Figure 1(a-c). Additionally, SWEAP's field of view (FOV) is designed to measure the solar wind and its aberration in the spacecraft frame (Kasper et al., 2016; Case et al., 2020; Whittlesey et al., 2020), leading to issues in sampling the planetary plasma.

\subsection{VGA1}

During VGA1 SWEAP/Solar Probe ANalyzers (SPAN) ion measurements did not capture the core proton distribution in its FOV, though electron measurements from SPAN were made. During VGA2, PSP was configured with the spacecraft boom in sunlight, in order to diagnose temperature dependence of the anomalous SCMx behavior, which unfortunately resulted in noisy SWEAP/Solar Probe Cup (SPC) measurements. However, SPAN measured distributions of both magnetosheath electrons and protons.

Figure 1 shows PSP's trajectory in the VSO $x-y$ plane during VGA1 (a) and VGA2 (b). Magnetic field data are shown in Figure 1(c-d). Five bow-shock cross- 
f1.jpg

Figure 1. (a-b) Trajectory of PSP during VGA1 and VGA2 in VSO $x-y$ plane. Black arrows show scaled plasma flow; purple arrows show measured magnetic field. (c-d) Vector magnetometer measurements for VGA1 and VGA2 (x, y, z/blue, green, red) with the magnitude (black). 
ings were recorded during VGA1. Figure 1(a) shows foreshock (FS) regions (blue, green, red), and magnetosheath (MS) regions (teal, yellow, black). Figure 2(a) shows vector magnetic time series for VGA1, with regions demarcated by dashed lines. Upstream quantities are $B_{0}=5.9 \mathrm{nT}, T_{p}=5.9 \mathrm{eV}, T_{e}=10.9 \mathrm{eV}, n_{p}=11 \mathrm{~cm}^{-3}, n_{e}=31 \mathrm{~cm}^{-3}$, $V_{s w}=410 \mathrm{~km} / \mathrm{s}$.

We focus on the downstream magnetosheath from 8:34:30-08:38:30, with $B_{0}=$ $12.7 \mathrm{nT}, T_{i}=11 \mathrm{eV}, T_{e}=14.45, \mathrm{eV} n_{p}=20, \mathrm{~cm}^{-3}, n_{e}=55 \mathrm{~cm}^{-3}$, and $V_{M S}=380 \mathrm{~km} / \mathrm{s}$. Magnetic coplanarity suggests quasi-parallel shock geometry, with a normal of $175^{\circ}$ (Paschmann \& Daly, 1998). Significant differences between $n_{e}$ and $n_{p}$ are observed both upstream and downstream; however the ratio $n_{e} / n_{i} \sim 2.7$ stays constant across the shock. Additionally, a cross shock density ratio, 1.8, is observed for both electrons and protons, suggesting that while error exists in the absolute measurement of density, the relative scaling is physical. Estimates for upstream $\beta_{p}$ range between 0.7-2.0; downstream $\beta_{p}$ ranges from 0.6-1.5.

Figure 2(b-c) shows trace power-spectra for the FS and MS. Largely non-powerlaw spectra are observed indicating significant wave activity and instabilities (Burgess et al., 2005). The MS fluctuations show power-law spectra, commonly associated with turbulence. Vertical lines show spacecraft frame frequencies corresponding to $k \rho_{i} \sim 1$ and $k \rho_{e} \sim 1$, assuming the Taylor hypothesis $k=2 \pi f / V_{s w}$. Magnetosheath kinetic spectra scale as $k^{-2.9}$, and no spectral break observed at ion-kinetic scales (e.g. $\left.k \rho_{i} \sim 1\right)$. The extension of kinetic range spectra into inertial range frequencies has been interpreted as the result of FLR effects (Uritsky et al., 2011); parallel shock dynamics likely affect plasma kinetics in this region, leading to the lack of an observed inertial range (Xiao et al., 2018). Sahraoui et al. (2006) attribute the extension of kinetic range scaling into fluid-scales with the presence of mirror modes. Spectral properties of the three MS regions are similar, though strong electron scale wave activity observed behind the first shock crossing (teal) is seemingly absent from other MS intervals.

\subsection{VGA2}

During VGA2, two (inbound and outbound) shock crossings occurred, Figure 2(d,e) shows separate FS and MS regions. Upstream parameters are $B_{0}=7.8 \mathrm{nT}$, $T_{e}=16 \mathrm{eV}, n_{p}=21 \mathrm{~cm}^{-3}, n_{e}=24 \mathrm{~cm}^{-3} V_{s} w=340 \mathrm{~km} / \mathrm{s}$, due to poor measurements of upstream $T_{i}$, we cannot report upstream $\beta_{i}$.

SPAN resolved the ion distribution in the downstream magnetosheath, characterized by: $B_{0}=14 \mathrm{nT}, T_{p}=92 \mathrm{eV}, n_{p}=15 \mathrm{~cm}^{-3}, n_{e}=57 \mathrm{~cm}^{-3}$. The significant difference between ion and electron densities is likely not physical: absolute ion-density is likely affected by FOV issues. There is decent agreement between $n_{e}$ and $n_{p}$ from SPC in the upstream solar wind; we set $n_{p}=n_{e}=57 \mathrm{~cm}^{-3}$. The downstream MS flow is $V_{M S}=276 \mathrm{~km} / \mathrm{s}$ and $V_{a}=B / \sqrt{2 \mu_{0} \rho}=40 \mathrm{~km} / \mathrm{s}$, such that the Taylor hypothesis is applicable for Alfvén waves. Magnetic coplanarity of the VGA2 bow-shock gives a shock normal of 115 degrees, quasi-perpendicular to the upstream field.

Figure 2(e) shows FS spectra with non-power-law scaling and significant wave activity; the MS spectra, Figure 2f shows power-law scaling. Figure $2(\mathrm{f}-\mathrm{g})$ shows $k^{-3.4}$ spectrum for scales between $k \rho_{i}=1$ and $k d_{e}=1$, with further steepening to an approximate $k^{-6.3}$ spectrum near electron scales. The steepening occurs at a frequency between $k \rho_{i}=1$ and $k d_{e}=1$, though there are uncertainties in the electron measurements. The observation of a secondary steepening at electron scales is consistent with observations in the terrestrial magnetosphere (Huang et al., 2014; Chen \& Boldyrev, 2017).

The spectral index of the MS spectra, $k^{-3.4}$, is significantly steeper than in VGA1, or what is typically associated with kinetic Alfvén wave (KAW) turbulence 
(Boldyrev \& Perez, 2012b; Zhao et al., 2016). Simulations can recover similarly steep spectra, though typically at low $\beta$ (Franci et al., 2015, 2016). At high $\beta$, increased damping may result in enhanced spectral steepening over the kinetic range (Howes et al., 2007, 2011). VGA2 shows an inertial-kinetic scale break around $k \rho_{i}=1$, which is not evident behind the quasi-parallel shock. The inertial range is possibly less steep than $k^{-5 / 3}$, thought due to the short interval it is difficult to measure with great confidence

Kinetic Alfvén wave turbulence is commonly associated with a $k^{-7 / 3}$ spectrum, with some variation from intermittency or damping (Howes et al., 2007; Boldyrev \& Perez, 2012b; Howes et al., 2011). The kinetic spectrum measured with $d_{i}<1 / k<d_{e}$ is significantly steeper than predictions of KAW turbulence (Schekochihin et al., 2009; Howes et al., 2011; Boldyrev \& Perez, 2012b; Franci et al., 2015, 2016; Zhao et al., 2016; Grošelj et al., 2018). Notably Rezeau et al. (1999), previously measured $k^{-3.4}$ scaling behind the terrestrial bow-shock. If the steep $k^{-3.4}$ spectrum is a signature of significant heating, the measured $T_{i} / T_{e}>1$ may indicate preferential ion heating through turbulent dissipation via Landau damping, which is observed in simulations at high $\beta$ (Kawazura et al., 2019).

\subsection{Temperature Anisotropy}

During VGA2, SPAN measured anisotropic temperatures, with $T_{\perp} / T_{\|} \sim 2$. At high $\beta$, significant $T_{\perp} / T_{\|}>1$ will drive mirror mode or Alfvén ion-cyclotron (AIC) instabilities (Gary, 1992; Hellinger et al., 2006; Bale et al., 2009). Figure 3 shows proton velocity distributions measured by SPAN-Ion during VGA2 in instrument coordinates. Instrumental FOV effects are highlighted by the cutoff in the $y$ direction. Bi-Maxwellian fits allow for computation of temperature anisotropies $T_{\perp} / T_{\|}$.

An alternative method of estimating the temperature anisotropy through diagonalizing the measured temperature moment tensor from SPAN verifies this measurement. The temperature tensor is rotated into a frame aligned with the magnetic field; assuming gyrotropy, there are enough degrees of freedom to calculate $T_{\perp}$ and $T_{\|}$from well-measured tensor components $\left(T_{x x}, T_{z z}, T_{x z}\right)$ without using the poorly-measured $y$ component. The independent methods of calculating temperature anisotropy provide similar results, and thus confidence in the measurement.

While turbulent heating significantly affects spectral indices, it's likely that the $T_{\perp} / T_{\|} \sim 2$ anisotropy plays a role in the kinetic cascade. Dwivedi et al. (2015) suggest that the kinetic scale spectra at Venus may relate to the growth of these instabilities in the magnetosheath. The growth of the AIC instability is associated with circularly polarized electromagnetic waves at ion scales (Verscharen et al., 2019). Analysis of polarization signatures reveals little significant circular polarization suggesting that a mirror instability may dominate; however, the angle between the mean field and the solar wind flow is $118^{\circ}$, such that quasi-parallel waves may be hard to identify (Bowen, Mallet, Huang, et al., 2020). Volwerk et al. (2008) previously reported mirror modes behind a quasi-perpendicular bow shock at Venus. At $T_{\perp} / T_{\|} \sim 2$ and $\beta \sim 10$, growth rates for mirror mode may be larg, e. g. as $0.1 \omega_{c}$ (Hellinger et al., 2006). For $f_{c i} \sim 1 \mathrm{~Hz}$, this corresponds to a growth rate of $\sim 10 \mathrm{~s}$. The presence of $\alpha$ particles and other heavy ions in the magnetosphere can affect instability growth rates (Chen et al., 2016; Verscharen et al., 2019); it has been suggested that heavy ions stabilize the AIC instability (Price et al., 1986). The steep kinetic range spectrum may result from the introduction of KAW with nonlinear interactions with driven non-propagating mirror mode structures. The mirror mode is commonly associated with anti-correlated magnetic and kinetic pressure; however, the SPAN measurement cadence is not sufficient to determine correlations at kinetic scales. 
f2.jpg

Figure 2. (a) Vector magnetic field measurements from VGA1. Color coded lines demarcate three foreshock regions (blue, green, orange) from three magneosheath regions (teal, yellow, black). (b,c) Color coded power-spectra for intervals shown in (a); dashed/dotted lines correspond to convected ion/electron gyroradius $k \rho_{i / e}$. Purple curve shows SCM sensitivity. (d) Vector magnetic field measurements from VGA2. (e) Power spectra from foreshock regions (blue,green, orange) and $k \rho_{i / e}$. (f) Magnetosheath spectra with convected ion/electron gyroradius $k \rho_{i / e}$ and inertial length $k d_{i / e}$. (e) Magnetosheath spectra from 10-100 Hz, showing electron scale steepening. 
f3. pdf

Figure 3. (a-c) Proton distributions from VGA2 magnetosheath observed by SPAN at three times in sensor $x-y$ plane. (d-f) Proton distributions from SPAN for VGA2 magnetosheath in sensor $x-z$ plane. The magnetic field, in Alfvén units, is shown as a black arrow. 
Sahraoui et al. (2006) discuss the mirror instability in the terrestrial magnetosheath, demonstrating non-propagating structures characteristic of the mirror mode; however they measure an energy spectrum similar to the canonical KAW $k^{-2.7}$ scaling, which extends into scales typically associated with the inertial range. The presence of these modes, and other instabilities, likely effects observed signatures of kinetic scale turbulence.

\section{Signatures of a Kinetic Cascade}

Systematically shallow spectra at inertial scales suggest that inertial range magnetosheath turbulence may not always form (Czaykowska et al., 2001; Alexandrova et al., 2008). Whether instabilities can drive kinetic scale turbulence in the absence of an inertial range cascade is an open question (Hadid et al., 2015). The higher order moments of distributions of turbulent fluctuations provide information regarding the development and dissipation of turbulence (Matthaeus et al., 2015; Tessein et al., 2013; Mallet et al., 2019; Bandyopadhyay et al., 2020).

Distributions of turbulent fluctuations are often characterized with statistical moments of increments, (Monin \& Yaglom, 1971, 1975; Dudok de Wit \& Krasnoselkikh, 1996; Sorriso-Valvo et al., 1999; Hnat et al., 2002; Kiyani et al., 2006). However, increments cannot resolve spectral scaling steeper than $k^{-3}$ (Frisch, 1995; Cho \& Lazarian, 2009). For scalings observed in the Venus magnetosheath, alternative measurements of fluctuation amplitudes, such as the continuous wavelet transform (CWT), are required to capture higher order properties of kinetic range turbulence (Farge, 1992; Farge \& Schneider, 2015; Kiyani et al., 2015).

$$
\tilde{B}(s, \tau)=\sum_{i=0}^{N-1} \psi\left(\frac{t_{i}-\tau}{s}\right) B_{j}\left(t_{i}\right) ;
$$

we use the Morlet wavelet

$$
\psi(\xi)=\pi^{-1 / 4} e^{-i \omega_{0} \xi} e^{\frac{-\xi^{2}}{2}},
$$

with $\omega_{0}=6$.

Figure 4(a-b) shows $\left\langle\sigma_{s}^{2}\right\rangle$ for VGA1 and VGA2. Figure 4(c-d) show the scale dependent kurtosis $\kappa=\left\langle\left|\tilde{B}^{4}\right|\right\rangle /\left\langle\sigma_{s}^{2}\right\rangle=$ computed for each wavelet scale. Increasing $\kappa$ is seen in both VGA1 and VGA2 at $f \gtrsim 10 \mathrm{~Hz}$.

Excluding outlier fluctuations at a given scale, conditioning, decreases effects of transients, e.g. those observed in VGA1 and VGA2 by Page (2020) and Goodrich (2020), on $\kappa$ (Kiyani et al., 2006). For each scale, wavelet coefficients with $\sigma^{2}>$ $F\left\langle\sigma^{2}\right\rangle$ are removed for $F=3,10,30,70,100$, and $\left\langle\sigma^{2}\right\rangle$ and $\kappa$ are recomputed. Large decreases in $\kappa$ are observed when removing outliers, while the power is not greatly affected. The conditioning has similar effects for both VGA1 and VGA2, indicating that though the spectral scalings differ, the scaling of kurtosis is similar. In both cases $\mathrm{F}=10$, removes approximately $1 \%$ of fluctuations in sub-ion scales, though the kurtosis remains larger than 3 (expected for Gaussian fluctuations). This indicates the presence of non-Gaussian fluctuations commonly associated with kinetic range turbulence (Kiyani et al., 2009; Hadid et al., 2015; Kiyani et al., 2015). Higher order moments can be difficult to compute accurately for finite sample lengths (Kiyani et al., 2006). Dudok de Wit (2004) suggest requiring explicit convergence of higher order moments, though they derive an approximate required number of samples given by $\log _{10} N-1$. For these 4 minute, $(N \sim 70000)$ records, $\log _{10}(N)-1=3.85$, suggesting that kurtosis may not be perfectly resolved. While our measurement of 
Figure 4. (a,b) CWT spectra $\left\langle\sigma^{2}\right\rangle$ for VGA1 and VGA2 (black); colors correspond to conditioned spectra. (c,d) Effect of conditioning on wavelet kurtosis for VGA1 and VGA2. (e-f) Percentage of clipped wavelet coefficients at each conditioning level.

kurtosis may lack accuracy, non-Gaussianity of kinetic scale fluctuations is evident in the distributions of wavelet coefficients (not shown). Hadid et al. (2015) show different scaling properties of higher order moments of turbulent amplitudes behind quasi-perpendicular and quasi-parallel shocks at Saturn, implying differences in the kinetic scale intermittency, but do not peform any conditioning.

\section{Summary}

We present measurements of kinetic scale turbulence in the Venusian magnetoheath behind both a quasi-perpendicular and quasi-parallel bow shock. A steep kinetic range spectrum is observed behind the quasi-perpendicular (VGA2) shock with a subion $k^{-3.4}$ scaling. Observation of significant temperature anisotropy $\left(T_{\perp} / T_{\|} \sim 2\right)$ in $\beta \sim 10$ plasma suggests that the mirror or Alfvén ion cyclotron instabilities are quite strong; the lack of observed circular polarization suggests a dominant mirror instability (Gary, 1992; Hellinger et al., 2006). The nonlinear generation of mirror modes (Southwood \& Kivelson, 1993) may increase nonlinear interaction rates at kinetic scales, steepening the cascade from typically observed $k^{-8 / 3}$ spectra (Huang et al., 2014; von Papen et al., 2014; Hadid et al., 2015; Chen \& Boldyrev, 2017). The steep spectra may also be associated with preferential ion heating at high $\beta$ (Kawazura et al., 2019). At $k d_{e}=1$ a secondary kinetic steepening is observed consistent with the observations of the terrestrial magnetosphere (Huang et al., 2014; Chen \& Boldyrev, 2017). Behind the quasi-parallel shock a $k^{-2.9}$ scaling occurs; no measurements of temperature anisotropy were available. Though spectral energy scaling varies between Venus encounters, the kurtosis in either case shows similar signatures of non-Gaussianity, indicating kinetic range developed turbulence. Our results highlight the importance of ion-scale instabilities in shaping kinetic turbulence in planetary environments.

\section{Acknowledgements}

Parker Solar Probe FIELDS and SWEAP instrumentation were developed under contract NNN06AA01C. PSP data is publicly available at NASA Space Physics Data Facility (SPDF) https://cdaweb.gsfc.nasa.gov/. FIELDS data is also hosted at sprg.ssl.berkeley.edu/data/psp/data/sci/fields/. Discussion of the merged SCM and MAG (SCaM) data may be found in (Bowen, Bale, et al., 2020).

\section{References}

Alexandrova, O. (2008). Solar wind vs. magnetosheath turbulence and Alfvén vortices. Nonlin. Proc. Geophys., 15, 95.

Alexandrova, O., Lacombe, C., \& Mangeney, A. (2008, November). Spectra and anisotropy of magnetic fluctuations in the Earth's magnetosheath: 
Cluster observations. Annales Geophysicae, 26(11), 3585-3596.

doi: 10.5194/angeo-26-3585-2008

Alexandrova, O., Lacombe, C., Mangeney, A., Grappin, R., \& Maksimovic, M. (2012, December). Solar Wind Turbulent Spectrum at Plasma Kinetic Scales. ApJ, 760 (2), 121. doi: 10.1088/0004-637X/760/2/121

Alexandrova, O., Saur, J., Lacombe, C., Mangeney, A., Mitchell, J., Schwartz, S. J., \& Robert, P. (2009, Oct). Universality of Solar-Wind Turbulent Spectrum from MHD to Electron Scales. Physical Review Letters, 103(16), 165003. doi: 10.1103/PhysRevLett.103.165003

Amerstorfer, U. V., Erkaev, N. V., Langmayr, D., \& Biernat, H. K. ～（2007, October). On Kelvin Helmholtz instability due to the solar wind interaction with unmagnetized planets. Planetary and Space Science, 55(12), 1811-1816. doi: 10.1016/j.pss.2007.01.015

Bale, S. D., Goetz, K., Harvey, P. R., Turin, P., Bonnell, J. W., Dudok de Wit, T., ... Wygant, J. R. (2016, December). The FIELDS Instrument Suite for Solar Probe Plus. Measuring the Coronal Plasma and Magnetic Field, Plasma Waves and Turbulence, and Radio Signatures of Solar Transients. Space Science Rev., 204, 49-82. doi: 10.1007/s11214-016-0244-5

Bale, S. D., Kasper, J. C., Howes, G. G., Quataert, E., Salem, C., \& Sundkvist, D. (2009, November). Magnetic Fluctuation Power Near Proton Temperature Anisotropy Instability Thresholds in the Solar Wind. PRL, $103(21), 211101$. doi: 10.1103/PhysRevLett.103.211101

Balikhin, M. A., Zhang, T. L., Gedalin, M., Ganushkina, N. Y., \& Pope, S. A. (2008, January). Venus Express observes a new type of shock with pure kinematic relaxation. GRL, 35(1), L01103. doi: 10.1029/2007GL032495

Bandyopadhyay, R., Matthaeus, W. H., Parashar, T. N., Chhiber, R., Chasapis, A., Ruffolo, D., ... Raouafi, N. (2020). Observations of energetic-particle population enhancements along intermittent structures near the sun from parker solar probe. The Astrophysical Jounral Supplemental Series, PSP special issue (in press). Retrieved from https://ui.adsabs.harvard.edu/abs/ 2019arXiv191203424B

Boldyrev, S., \& Perez, J. C. (2012a, Oct). Spectrum of Kinetic-Alfvén Turbulence. ApJ, 758, L44. doi: 10.1088/2041-8205/758/2/L44

Boldyrev, S., \& Perez, J. C. (2012b, Oct). Spectrum of Kinetic-Alfvén Turbulence. The Astrophysical Journal Letters, 758(2), L44. doi: 10.1088/2041-8205/758/ $2 / \mathrm{L} 44$

Bowen, T. A., Bale, S. D., Bonnell, J. W., Dudok de Wit, T., Goetz, K., Goodrich, K., ... Szabo, A. (2020, May). A Merged Search-Coil and Fluxgate Magnetometer Data Product for Parker Solar Probe FIELDS. Journal of Geophysical Research (Space Physics), 125(5), e27813. doi: 10.1029/2020JA027813

Bowen, T. A., Mallet, A., Bale, S. D., Bonnell, J. W., Case, A. W., Chandran, B. D. G., .. Whittlesey, P. (2020, July). Constraining Ion-Scale Heating and Spectral Energy Transfer in Observations of Plasma Turbulence. PRL, 125(2), 025102. doi: 10.1103/PhysRevLett.125.025102

Bowen, T. A., Mallet, A., Huang, J., Klein, K. G., Malaspina, D. M., Stevens, M., ... Whittlesey, P. (2020, February). Ion-scale Electromagnetic Waves in the Inner Heliosphere. ApJS, 246(2), 66. doi: 10.3847/1538-4365/ab6c65

Bruno, R., \& Carbone, V. (2005, Sep 20). The solar wind as a turbulence laboratory. Living Reviews in Solar Physics, 2(1), 4. Retrieved from http://doi .org/10.12942/lrsp-2005-4 doi: 10.12942/lrsp-2005-4

Burgess, D., Lucek, E. A., Scholer, M., Bale, S. D., Balikhin, M. A., Balogh, A., ... Walker, S. N. (2005, June). Quasi-parallel Shock Structure and Processes. Space Science Rev., 118(1-4), 205-222. doi: 10.1007/s11214-005-3832-3

Case, A. W., Kasper, J. C., Stevens, M. L., Korreck, K. E., Paulson, K., Daigneau, P., .. Martinović, M. M. (2020, February). The Solar Probe Cup on the 
Parker Solar Probe. ApJS, 246(2), 43. doi: 10.3847/1538-4365/ab5a7b

Chen, C., Boldyrev, S., Xia, Q., \& Perez, J. (2013). Nature of subproton scale turbulence in the solar wind. Physical review letters, 110(22), 225002.

Chen, C. H. K. (2016, December). Recent progress in astrophysical plasma turbulence from solar wind observations. Journal of Plasma Physics, 82(6), 535820602. doi: 10.1017/S0022377816001124

Chen, C. H. K., \& Boldyrev, S. (2017, June). Nature of Kinetic Scale Turbulence in the Earth's Magnetosheath. ApJ, 842(2), 122. doi: 10.3847/1538-4357/ aa74e0

Chen, C. H. K., Horbury, T. S., Schekochihin, A. A., Wicks, R. T., Alexandrova, O., \& Mitchell, J. (2010, Jun). Anisotropy of Solar Wind Turbulence between Ion and Electron Scales. Physical Review Letters, 104 (25), 255002. doi: 10.1103/PhysRevLett.104.255002

Chen, C. H. K., Matteini, L., Schekochihin, A. A., Stevens, M. L., Salem, C. S., Maruca, B. A., ... Bale, S. D. (2016, July). Multi-species Measurements of the Firehose and Mirror Instability Thresholds in the Solar Wind. ApJL, 825(2), L26. doi: 10.3847/2041-8205/825/2/L26

Chen, C. H. K., Sorriso-Valvo, L., Šafránková, J., \& Němeček, Z. (2014, Jul). Intermittency of Solar Wind Density Fluctuations From Ion to Electron Scales. The Astrophysical Journal Letters, 789(1), L8. doi: 10.1088/2041-8205/789/1/L8

Chhiber, R., Chasapis, A., Bandyopadhyay, R., Parashar, T. N., Matthaeus, W. H., Maruca, B. A., ... Gershman, D. J. (2018, December). Higher-Order Turbulence Statistics in the Earth's Magnetosheath and the Solar Wind Using Magnetospheric Multiscale Observations. Journal of Geophysical Research (Space Physics), 123(12), 9941-9954. doi: 10.1029/2018JA025768

Cho, J., \& Lazarian, A. (2009, August). Simulations of Electron Magnetohydrodynamic Turbulence. ApJ, 701 (1), 236-252. doi: 10.1088/0004-637X/701/1/236

Coleman, P. J. (1968). Turbulence, viscosity, and dissipation in the solar wind plasma. The Astrophysical Journal, 153, 371-388. doi: 10.1086/149674

Czaykowska, A., Bauer, T. M., Treumann, R. A., \& Baumjohann, W. (2001, March). Magnetic field fluctuations across the Earth's bow shock. Annales Geophysicae, 19(3), 275-287. doi: 10.5194/angeo-19-275-2001

Delva, M., Bertucci, C., Volwerk, M., Lundin, R., Mazelle, C., \& Romanelli, N. (2015, January). Upstream proton cyclotron waves at Venus near solar maximum. Journal of Geophysical Research (Space Physics), 120(1), 344-354. doi: 10.1002/2014JA020318

Dudok de Wit, T. (2004, November). Can high-order moments be meaningfully estimated from experimental turbulence measurements? PRE, 70(5), 055302. doi: 10.1103/PhysRevE.70.055302

Dudok de Wit, T., \& Krasnoselkikh, V. V. (1996, January). Non-Gaussian statistics in space plasma turbulence: fractal properties and pitfalls\&lt;/a\&gt;. Nonlinear Processes in Geophysics, 3(4), 262-273. doi: 10.5194/npg-3-262-1996

Dwivedi, N. K., Schmid, D., Narita, Y., Kovács, P., Vörös, Z., Delva, M., \& Zhang, T. (2015, August). Statistical investigation on the power-law behavior of magnetic fluctuations in the Venusian magnetosheath. Earth, Planets, and Space, 67, 137. doi: 10.1186/s40623-015-0308-x

Farge, M. (1992, January). Wavelet transforms and their applications to turbulence. Annual Review of Fluid Mechanics, 24, 395-457. doi: 10.1146/annurev.fl .24 .010192 .002143

Farge, M., \& Schneider, K. (2015, December). Wavelet transforms and their applications to MHD and plasma turbulence: a review. Journal of Plasma Physics, 81(6), 435810602. doi: 10.1017/S0022377815001075

Fox, N. J., Velli, M. C., Bale, S. D., Decker, R., Driesman, A., Howard, R. A., ... Szabo, A. (2016, Dec 01). The solar probe plus mission: Humanity's first visit to our star. Space Science Reviews, 204(1), 7-48. Retrieved from https:// 
doi.org/10.1007/s11214-015-0211-6 doi: 10.1007/s11214-015-0211-6

Franci, L., Landi, S., Matteini, L., Verdini, A., \& Hellinger, P. (2015, Oct). Highresolution Hybrid Simulations of Kinetic Plasma Turbulence at Proton Scales. The Astrophysical Journal, 812(1), 21. doi: 10.1088/0004-637X/812/1/21

Franci, L., Landi, S., Matteini, L., Verdini, A., \& Hellinger, P. (2016, Dec). Plasma Beta Dependence of the Ion-scale Spectral Break of Solar Wind Turbulence: High-resolution 2D Hybrid Simulations. The Astrophysical Journal, 833(1), 91. doi: $10.3847 / 1538-4357 / 833 / 1 / 91$

Frisch, U. (1995). Turbulence.

Futaana, Y., Stenberg Wieser, G., Barabash, S., \& Luhmann, J. G. ～(2017, November). Solar Wind Interaction and Impact on the Venus Atmosphere. SSR, 212(3-4), 1453-1509. doi: 10.1007/s11214-017-0362-8

Gary, S. P. (1992, June). The mirror and ion cyclotron anisotropy instabilities. JGR, 97(A6), 8519-8529. doi: 10.1029/92JA00299

Golbraikh, E., Gedalin, M., Balikhin, M., \& Zhang, T. L. (2013, April). Large amplitude nonlinear waves in Venus magnetosheath. Journal of Geophysical Research (Space Physics), 118(4), 1706-1710. doi: 10.1002/jgra.50094

Goodrich, e. a., K.A. (2020). Electron Holes at Venus. GRL, This Issue.

Grošelj, D., Mallet, A., Loureiro, N. F., \& Jenko, F. (2018, March). Fully Kinetic Simulation of 3D Kinetic Alfvén Turbulence. PRL, 120(10), 105101. doi: 10 .1103/PhysRevLett.120.105101

Hadid, L. Z., Sahraoui, F., Kiyani, K. H., Retinò, A., Modolo, R., Canu, P., ... Dougherty, M. K. (2015, November). Nature of the MHD and Kinetic Scale Turbulence in the Magnetosheath of Saturn: Cassini Observations. ApJL, 813(2), L29. doi: 10.1088/2041-8205/813/2/L29

Hellinger, P., Trávníček, P., Kasper, J. C., \& Lazarus, A. J. $\quad$ (2006, May). Solar wind proton temperature anisotropy: Linear theory and WIND/SWE

observations. Geophysical Research Letters, 33, L09101. Retrieved from http://adsabs.harvard.edu/abs/2006GeoRL. 33.9101H doi: 10.1029/2006GL025925

Hnat, B., Chapman, S. C., Rowlands, G., Watkins, N. W., \& Farrell, W. M. (2002, May). $\quad$ Finite size scaling in the solar wind magnetic field energy density as seen by WIND. GRL, 29(10), 1446. doi: 10.1029/2001GL014587

Howes, G. G., Cowley, S. C., Dorland, W., Hammett, G. W., Quataert, E., \& Schekochihin, A. A. (2007, August). Dissipation-scale turbulence in the solar wind. In D. Shaikh \& G. P. Zank (Eds.), Turbulence and nonlinear processes in astrophysical plasmas (Vol. 932, p. 3-8). doi: 10.1063/1.2778938

Howes, G. G., Tenbarge, J. M., Dorland, W., Quataert, E., Schekochihin, A. A., Numata, R., \& Tatsuno, T. (2011, Jul). Gyrokinetic Simulations of Solar Wind Turbulence from Ion to Electron Scales. Physical Review Letters, 107 (3), 035004. doi: 10.1103/PhysRevLett.107.035004

Huang, S. Y., Hadid, L. Z., Sahraoui, F., Yuan, Z. G., \& Deng, X. H. (2017, February). On the Existence of the Kolmogorov Inertial Range in the Terrestrial Magnetosheath Turbulence. ApJL, 836(1), L10. doi: 10.3847/2041-8213/836/ 1/L10

Huang, S. Y., Sahraoui, F., Deng, X. H., He, J. S., Yuan, Z. G., Zhou, M., .. Fu, H. S. (2014, July). Kinetic Turbulence in the Terrestrial Magnetosheath: Cluster Observations. ApJL, 789(2), L28. doi: 10.1088/2041-8205/789/2/L28

Huang, S. Y., Wang, Q. Y., Sahraoui, F., Yuan, Z. G., Liu, Y. J., Deng, X. H., ... Zhang, J. (2020, March). Analysis of Turbulence Properties in the Mercury Plasma Environment Using MESSENGER Observations. $\quad$ ApJ, 891(2), 159. doi: $10.3847 / 1538-4357 /$ ab7349

Kasper, J. C., Abiad, R., Austin, G., Balat-Pichelin, M., Bale, S. D., Belcher, J. W., ... Zank, G. (2016, Dec 01). Solar wind electrons alphas and protons (sweap) investigation: Design of the solar wind and coronal plasma 
instrument suite for solar probe plus. Space Science Reviews, 204(1), 131186. Retrieved from https://doi.org/10.1007/s11214-015-0206-3 doi: 10.1007/s11214-015-0206-3

Kawazura, Y., Barnes, M., \& Schekochihin, A. A. (2019, January). Thermal disequilibration of ions and electrons by collisionless plasma turbulence. Proceedings of the National Academy of Science, 116(3), 771-776. doi: 10.1073/pnas .1812491116

Kiyani, K. H., Chapman, S. C., \& Hnat, B. (2006, November). Extracting the scaling exponents of a self-affine, non-Gaussian process from a finite-length time series. PRE, 74 (5), 051122. doi: 10.1103/PhysRevE.74.051122

Kiyani, K. H., Chapman, S. C., Khotyaintsev, Y. V., Dunlop, M. W., \& Sahraoui, F. (2009, Aug). Global Scale-Invariant Dissipation in Collisionless Plasma Turbulence. Physical Review Letters, 103(7), 075006. doi: 10.1103/ PhysRevLett.103.075006

Kiyani, K. H., Osman, K. T., \& Chapman, S. C. $\quad$ (2015, Apr). Dissipation and heating in solar wind turbulence: from the macro to the micro and back again. Philosophical Transactions of the Royal Society of London Series A, 373(2041), 20140155-20140155. doi: 10.1098/rsta.2014.0155

Kletzing, C. A., Kurth, W. S., Acuna, M., MacDowall, R. J., Torbert, R. B., Averkamp, T., ... Tyler, J. (2013, November). The Electric and Magnetic Field Instrument Suite and Integrated Science (EMFISIS) on RBSP. $\quad S S R$, 179(1-4), 127-181. doi: 10.1007/s11214-013-9993-6

Leamon, R. J., Smith, C. W., Ness, N. F., Matthaeus, W. H., \& Wong, H. K. (1998, March). Observational constraints on the dynamics of the interplanetary magnetic field dissipation range. $\quad J G R, 103(\mathrm{~A} 3), 4775-4788 . \quad$ doi: 10.1029/97JA03394

Malaspina, D. M., Ergun, R. E., Bolton, M., Kien, M., Summers, D., Stevens, K., ... Goetz, K. (2016, June). The Digital Fields Board for the FIELDS instrument suite on the Solar Probe Plus mission: Analog and digital signal processing. Journal of Geophysical Research (Space Physics), 121, 5088-5096. doi: 10.1002/2016JA022344

Mallet, A., Klein, K. G., Chand ran, B. D. G., Grošelj, D., Hoppock, I. W., Bowen, T. A., ... Bale, S. D. (2019, Jun). Interplay between intermittency and dissipation in collisionless plasma turbulence. Journal of Plasma Physics, 85(3), 175850302. doi: $10.1017 /$ S0022377819000357

Matthaeus, W. H., \& Goldstein, M. L. (1982). Measurement of the rugged invariants of magnetohydrodynamic turbulence in the solar wind. J. Geophys. Res., 87(A8), 6011-6028. Retrieved from http://dx.doi.org/10.1029/ JA087iA08p06011 doi: 10.1029/JA087iA08p06011

Matthaeus, W. H., Wan, M., Servidio, S., Greco, A., Osman, K. T., Oughton, S., \& Dmitruk, P. (2015). Intermittency, nonlinear dynamics and dissipation in the solar wind and astrophysical plasmas. Philosophical Transactions of the Royal Society of London A: Mathematical, Physical and Engineering Sciences, 373(2041). Retrieved from https://doi.org/10.1098/rsta.2014.0154 doi: 10.1098/rsta.2014.0154

McFadden, J. P., Carlson, C. W., Larson, D., Ludlam, M., Abiad, R., Elliott, B., ... Angelopoulos, V. (2008, December). The THEMIS ESA Plasma Instrument and In-flight Calibration. SSR, 141(1-4), 277-302. doi: 10.1007/s11214-008-9440-2

Monin, A. S., \& Yaglom, A. M. (1971). Statistical fluid mechanics, vol. 1. Cambridge, Mass.: MIT Press.

Monin, A. S., \& Yaglom, A. M. (1975). Statistical fluid mechanics, vol. 2. Cambridge, Mass.: MIT Press. Retrieved from https://ui.adsabs.harvard.edu/ abs/1971sfmm.book.....M

Page, e. a., B. (2020). Electron Holes at Venus. GRL, This Issue. 
Paschmann, G., \& Daly, P. W. (1998). Analysis methods for multi-spacecraft data. issi scientific reports series sr-001, esa/issi, vol. 1. isbn 1608-280x, $1998 . \quad$ ISSI Scientific Reports Series, 1. Retrieved from http://hdl.handle.net/11858/ 00-001M-0000-0014-D93A-D

Pope, S. A., Balikhin, M. A., Zhang, T. L., Fedorov, A. O., Gedalin, M., \& Barabash, S. (2009, April). Giant vortices lead to ion escape from Venus and re-distribution of plasma in the ionosphere. $\quad G R L, 36(7)$, L07202. doi: 10.1029/2008GL036977

Price, C. P., Swift, D. W., \& Lee, L. C. (1986, January). Numerical simulation of nonoscillatory mirror waves at the Earth's magnetosheath. JGR, 91(A1), 101112. doi: 10.1029/JA091iA01p00101

Pulupa, M., Bale, S. D., Bonnell, J. W., Bowen, T. A., Carruth, N., Goetz, K., ... Sundkvist, D. (2017, March). The Solar Probe Plus Radio Frequency Spectrometer: Measurement requirements, analog design, and digital signal processing. Journal of Geophysical Research (Space Physics), 122(3), 2836-2854. doi: 10.1002/2016JA023345

Rezeau, L., Belmont, G., Cornilleau-Wehrlin, N., Reberac, F., \& Briand, C. (1999, January). Spectral Law and Polarization Properties of the LowFrequency Waves at the Magnetopause. GRL, 26(6), 651-654. doi: 10.1029/1999GL900060

Ruhunusiri, S., Halekas, J. S., Espley, J. R., Mazelle, C., Brain, D., Harada, Y., ... Howes, G. G. (2017, January). Characterization of turbulence in the Mars plasma environment with MAVEN observations. Journal of Geophysical Research (Space Physics), 122(1), 656-674. doi: 10.1002/2016JA023456

Russell, C. T., Leinweber, H., Hart, R. A., Wei, H. Y., Strangeway, R. J., \& Zhang, T. L. (2013, November). Venus Express observations of ULF and ELF waves in the Venus ionosphere: Wave properties and sources. Icarus, 226(2), 1527-1537. doi: 10.1016/j.icarus.2013.08.019

Russell, C. T., Mayerberger, S. S., \& Blanco-Cano, X. (2006, January). Proton cyclotron waves at Mars and Venus. Advances in Space Research, 38(4), 745-751. doi: $10.1016 /$ j.asr.2005.02.091

Sahraoui, F., Belmont, G., Rezeau, L., Cornilleau-Wehrlin, N., Pinçon, J. L., \& Balogh, A. (2006, February). Anisotropic Turbulent Spectra in the Terrestrial Magnetosheath as Seen by the Cluster Spacecraft. $\quad P R L, 96(7), 075002 . \quad$ doi: 10.1103/PhysRevLett.96.075002

Sahraoui, F., Goldstein, M. L., Belmont, G., Canu, P., \& Rezeau, L.

$(2010$, Sep). $\quad$ Three Dimensional Anisotropic k Spectra of Turbulence at Subproton Scales in the Solar Wind. Physical Review Letters, 105(13), 131101. doi: 10.1103/PhysRevLett.105.131101

Sahraoui, F., Goldstein, M. L., Robert, P., \& Khotyaintsev, Y. V.

(2009, Jun). Evidence of a Cascade and Dissipation of Solar-Wind Turbulence at the Electron Gyroscale. Physical Review Letters, 102(23), $231102 . \quad$ doi: 10.1103/PhysRevLett.102.231102

Sahraoui, F., Huang, S. Y., Belmont, G., Goldstein, M. L., Rétino, A., Robert, P., \& De Patoul, J. (2013, November). Scaling of the Electron Dissipation Range of Solar Wind Turbulence. ApJ, 777 (1), 15. doi: 10.1088/0004-637X/777/1/15

Saur, J. (2004, February). Turbulent Heating of Jupiter's Middle Magnetosphere. ApJL, 602(2), L137-L140. doi: 10.1086/382588

Schekochihin, A. A., Cowley, S. C., Dorland, W., Hammett, G. W., Howes, G. G., Quataert, E., \& Tatsuno, T. (2009, May). Astrophysical Gyrokinetics: Kinetic and Fluid Turbulent Cascades in Magnetized Weakly Collisional Plasmas. The Astrophysical Journal Supplement, 182, 310-377. doi: $10.1088 / 0067-0049 / 182 / 1 / 310$

Sorriso-Valvo, L., Carbone, V., Veltri, P., Consolini, G., \& Bruno, R. January). Intermittency in the solar wind turbulence through probabil- 
ity distribution functions of fluctuations. 10.1029/1999GL900270

Southwood, D. J., \& Kivelson, M. G. (1993, June). Mirror instability. I - Physical mechanism of linear instability. JGR, $98(\mathrm{~A} 6), 9181-9187 . \quad$ doi: 10.1029/ 92JA02837

Strangeway, R. J. (2004, January). Plasma waves and electromagnetic radiation at Venus and Mars. Advances in Space Research, 33(11), 1956-1967. doi: 10 $.1016 /$ j.asr.2003.08.040

Sundkvist, D., Retino, A., Vaivads, A., \& Bale, S. D. $\quad$ (2007). Dissipation in turbulent plasma due to reconnection in thin current sheets. Phys. Rev. Lett., 99, 025004. Retrieved from https://doi.org/10.1103/PhysRevLett.99.025004 doi: 10.1103/PhysRevLett.99.025004

Tao, C., Sahraoui, F., Fontaine, D., Patoul, J., Chust, T., Kasahara, S., \& Retinò, A. (2015, April). Properties of Jupiter's magnetospheric turbulence observed by the Galileo spacecraft. Journal of Geophysical Research (Space Physics), 120(4), 2477-2493. doi: 10.1002/2014JA020749

Tessein, J. A., Matthaeus, W. H., Wan, M., Osman, K. T., Ruffolo, D., \& Giacalone, J. (2013). Association of suprathermal particles with coherent structures and shocks. The Astrophysical Journal Letters, 776(1), L8. Retrieved from http://stacks.iop.org/2041-8205/776/i=1/a=L8 doi: 10.1088/2041-8205/776/1/L8

Uritsky, V. M., Slavin, J. A., Khazanov, G. V., Donovan, E. F., Boardsen, S. A., Anderson, B. J., \& Korth, H. (2011, September). Kinetic-scale magnetic turbulence and finite Larmor radius effects at Mercury. Journal of Geophysical Research (Space Physics), 116(A9), A09236. doi: 10.1029/2011JA016744

Verscharen, D., Klein, K. G., \& Maruca, B. A. (2019, December). The multi-scale nature of the solar wind. Living Reviews in Solar Physics, 16(1), 5. doi: 10 .1007/s41116-019-0021-0

Volwerk, M., Schmid, D., Tsurutani, B. T., Delva, M., Plaschke, F., Narita, Y., ... Glassmeier, K.-H. (2016, November). Mirror mode waves in Venus's magnetosheath: solar minimum vs. solar maximum. Annales Geophysicae, 34 (11), 1099-1108. doi: 10.5194/angeo-34-1099-2016

Volwerk, M., Zhang, T. L., Delva, M., Vörös, Z., Baumjohann, W., \& Glassmeier, K. H. (2008, December). Mirror-mode-like structures in Venus' induced magnetosphere. Journal of Geophysical Research (Planets), 113(15), E00B16. doi: 10.1029/2008JE003154

von Papen, M., Saur, J., \& Alexandrova, O. ～(2014, April). Turbulent magnetic field fluctuations in Saturn's magnetosphere. Journal of Geophysical Research (Space Physics), 119(4), 2797-2818. doi: 10.1002/2013JA019542

Vörös, Z., Zhang, T. L., Leaner, M. P., Volwerk, M., Delva, M., \& Baumjohann, W. (2008, December). Intermittent turbulence, noisy fluctuations, and wavy structures in the Venusian magnetosheath and wake. Journal of Geophysical Research (Planets), 113(E12), E00B21. doi: 10.1029/2008JE003159

Vörös, Z., Zhang, T. L., Leubner, M. P., Volwerk, M., Delva, M., Baumjohann, W., \& Kudela, K. (2008, June). Magnetic fluctuations and turbulence in the Venus magnetosheath and wake. GRL, 35(11), L11102. doi: 10.1029/2008GL033879

Walker, S. N., Balikhin, M. A., Zhang, T. L., Gedalin, M. E., Pope, S. A., Dimmock, A. P., \& Fedorov, A. O. (2011, January). Unusual nonlinear waves in the Venusian magnetosheath. Journal of Geophysical Research (Space Physics), 116(A1), A01215. doi: 10.1029/2010JA015916

Whittlesey, P. L., Larson, D. E., Kasper, J. C., Halekas, J., Abatcha, M., Abiad, R., .. Verniero, J. L. (2020, February). The Solar Probe ANalyzers-Electrons on the Parker Solar Probe. ApJS, 246(2), 74. doi: 10.3847/1538-4365/ab7370

Wolff, R. S., Goldstein, B. E., \& Yeates, C. M. (1980, December). The onset and development of Kelvin-Helmholtz instability at the Venus ionopause. JGR, 85, 
7697-7707. doi: 10.1029/JA085iA13p07697

Wygant, J. R., Bonnell, J. W., Goetz, K., Ergun, R. E., Mozer, F. S., Bale, S. D., ... Tao, J. B. (2013, November). The Electric Field and Waves Instruments on the Radiation Belt Storm Probes Mission. SSR, 179(1-4), 183-220. doi: 10.1007/s11214-013-0013-7

Xiao, S. D., Zhang, T. L., \& Vörös, Z. (2018, October). Magnetic Fluctuations and Turbulence in the Venusian Magnetosheath Downstream of Different Types of Bow Shock. Journal of Geophysical Research (Space Physics), 123(10), 8219-8226. doi: 10.1029/2018JA025250

Xiao, S. D., Zhang, T. L., Vörös, Z., Wu, M. Y., Wang, G. Q., \& Chen, Y. Q. (2020, February). Turbulence Near the Venusian Bow Shock: Venus Express Observations. Journal of Geophysical Research (Space Physics), 125(2), e27190. doi: 10.1029/2019JA027190

Zhang, T. L., Delva, M., Baumjohann, W., Auster, H. U., Carr, C., Russell, C. T., ... Lebreton, J. P. $\quad$ (2007, November). Little or no solar wind enters Venus' atmosphere at solar minimum. Nature, 450(7170), 654-656. doi: 10.1038 /nature06026

Zhao, J. S., Voitenko, Y. M., Wu, D. J., \& Yu, M. Y. (2016, January). Kinetic Alfvén turbulence below and above ion cyclotron frequency. Journal of Geophysical Research (Space Physics), 121(1), 5-18. doi: 10.1002/2015JA021959 

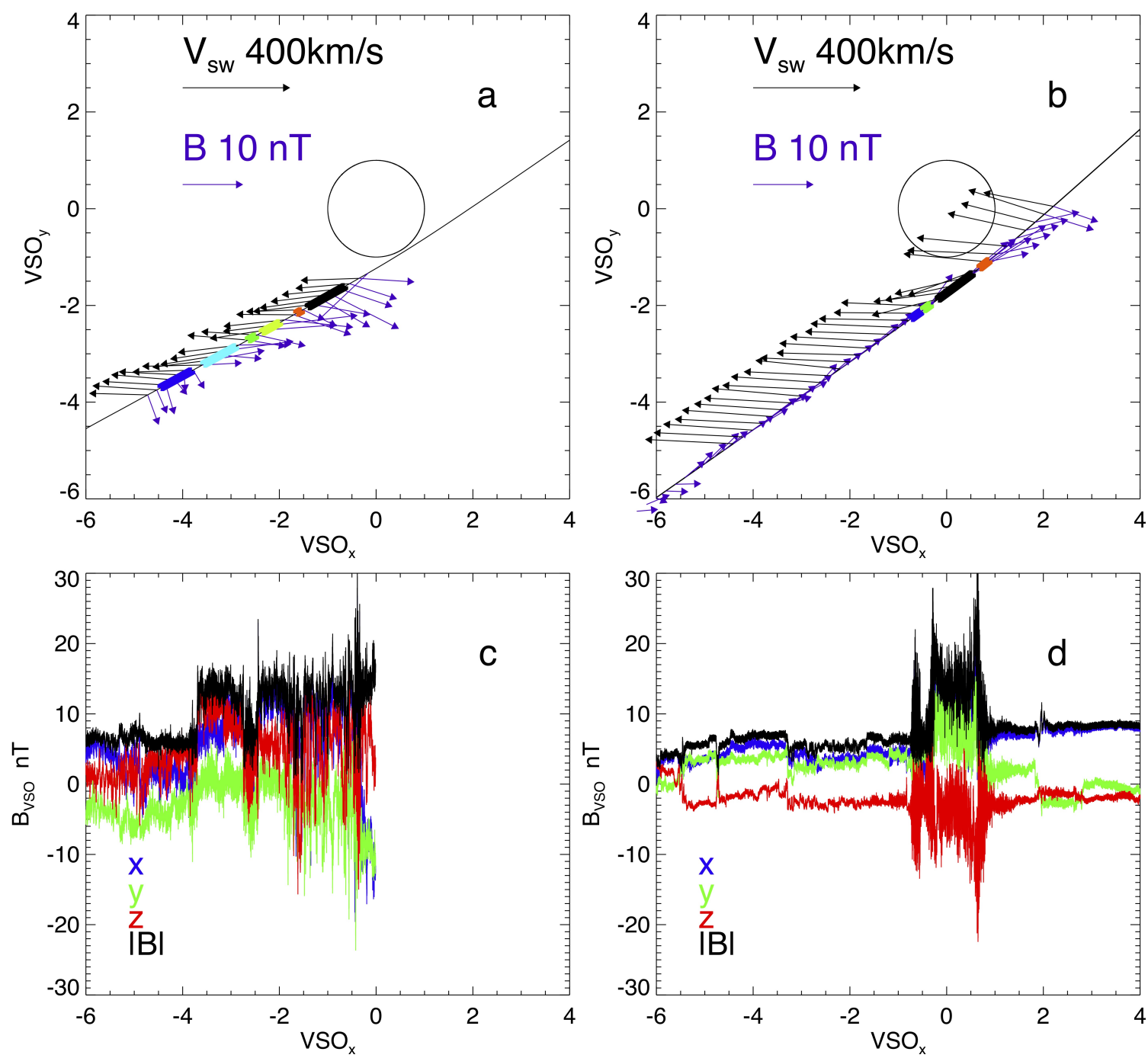

This article is protected by copyright. All rights reserved. 

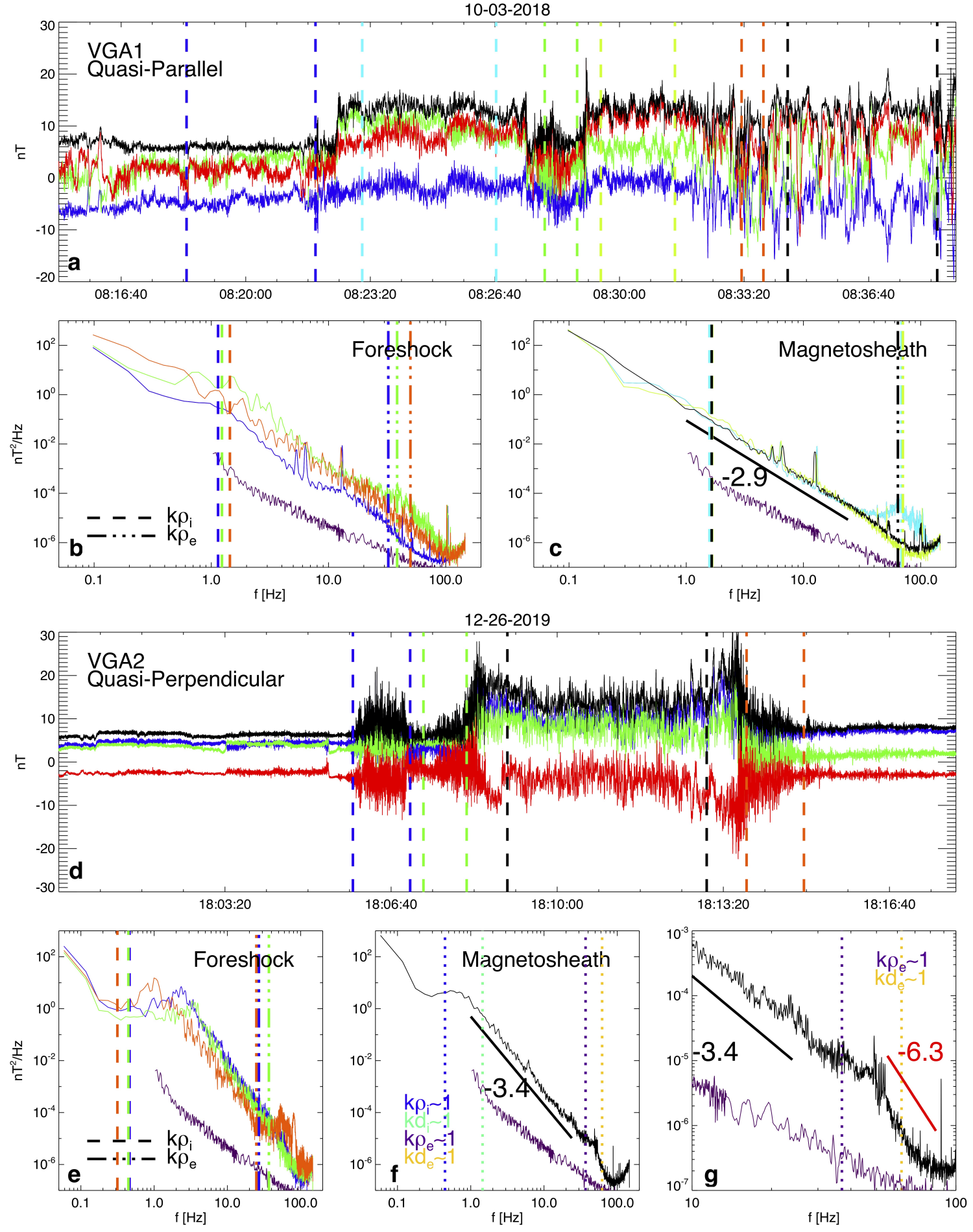

This article is protected by copyright. All rights reserved. 

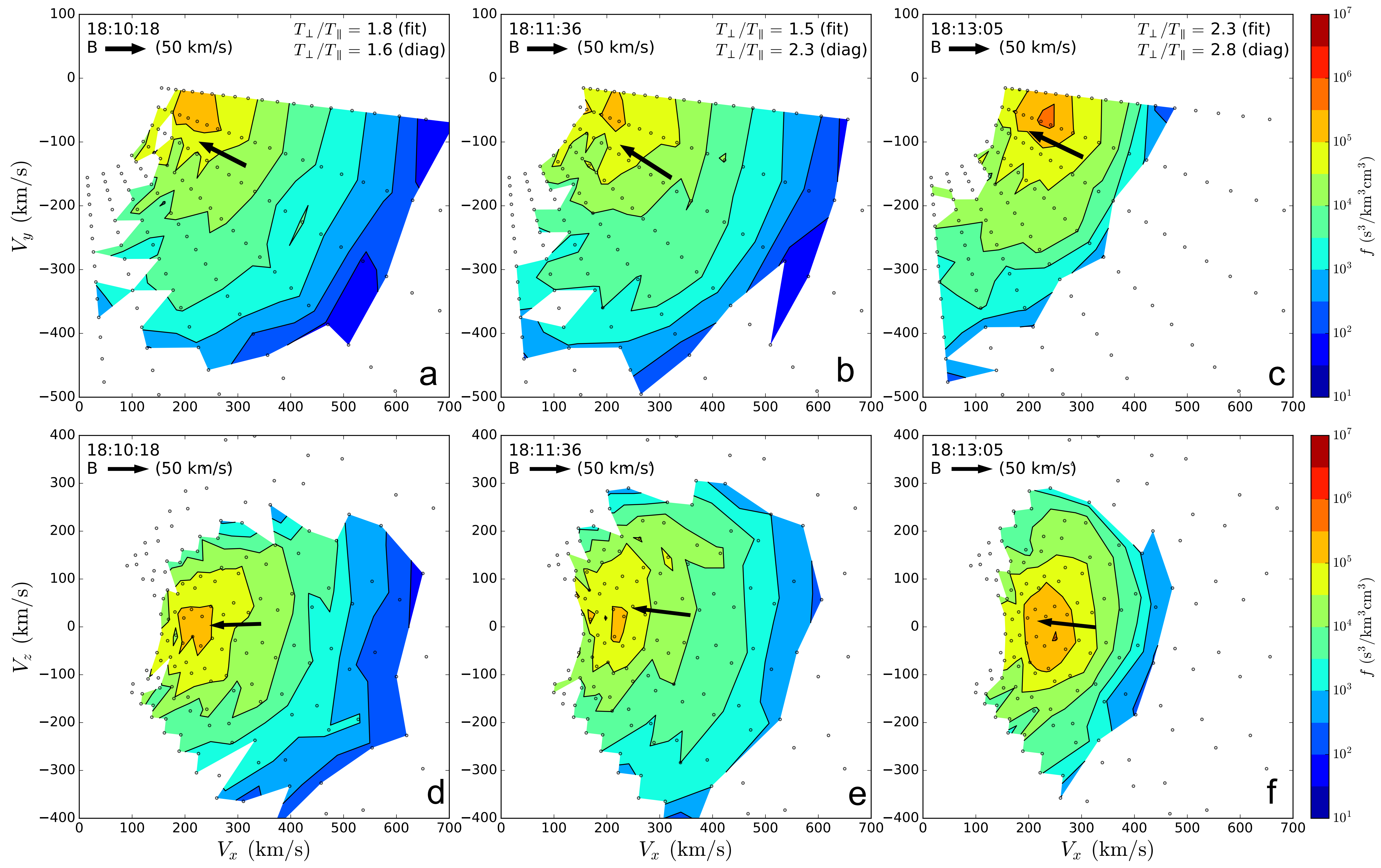
